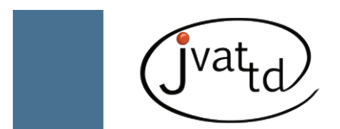

\title{
Embryotoxicity following repetitive maternal exposure to scorpion venom
}

Hmed BN (1), Riadh B (2), Serria H (1), Kamel J (3), Khaled Z (1)

(1) Laboratory of Pharmacology, Sfax Faculty of Medicine, Sfax, Tunisia; (2) Laboratory of Histology and Embryology, Sfax Faculty of Medicine, Sfax, Tunisia; (3) Laboratory of Biochemistry, Sfax Faculty of Medicine, Sfax, Tunisia.

\begin{abstract}
Although it is a frequent accident in a few countries, scorpion envenomation during pregnancy remains scarcely studied. In the present study, the effects of repetitive maternal exposure to Buthus occitanus tunetanus venom are investigated and its possible embryotoxic consequences on rats. Primigravid rats received a daily intraperitoneal dose of $1 \mathrm{~mL} / \mathrm{kg}$ of saline solution or $300 \mu \mathrm{g} / \mathrm{kg}$ of crude scorpion venom, from the $7^{\text {th }}$ to the $13^{\text {th }}$ day of gestation. On the $21^{\text {st }}$ day, the animals were deeply anesthetized using diethylether. Then, blood was collected for chemical parameter analysis. Following euthanasia, morphometric measurements were carried out. The results showed a significant increase in maternal heart and lung absolute weights following venom treatment. However, the mean placental weight per rat was significantly diminished. Furthermore, blood urea concentration was higher in exposed rats $(6.97 \pm 0.62 \mathrm{mmol} / \mathrm{L})$ than in those receiving saline solution $(4.94 \pm 0.90 \mathrm{mmol} / \mathrm{L})$. Many organs of venom-treated rat fetuses (brain, liver, kidney and spleen) were smaller than those of controls. On the contrary, fetal lungs were significantly heavier in fetuses exposed to venom $(3.2 \pm 0.4 \mathrm{~g})$ than in the others $(3.0 \pm 0.2 \mathrm{~g})$. Subcutaneous blood clots, microphthalmia and total body and tail shortening were also observed in venom-treated fetuses. It is concluded that scorpion envenomation during pregnancy potentially causes intrauterine fetal alterations and growth impairment.
\end{abstract}

Key words: prenatal repetitive exposure, scorpions, venom, rats.

\section{INTRODUCTION}

Scorpion envenoming is a widespread accident in tropical and subtropical regions. Its toxicological patterns had been thoroughly studied. They range from local benign symptoms to much worse manifestations including cardiorespiratory distress. Hemodynamic fluctuations, respiratory dysfunction, renal failure, hyperglycemia, lipolysis, free radical release, electrolytes and acid-base imbalance and other disorders can be observed following a scorpion bite (1). In addition, scorpion envenoming could result in hormonal imbalance such as estradiol and prostaglandin release in different animal models $(2,3)$. The mainly pathological mechanisms are direct or indirect consequences of the activities of various biologically active scorpion polypeptides. These peptides essentially comprise toxins that modify the membrane cell potential. Albeit scorpion sting is a frequent accident that causes approximately one million human envenomations each year, its incidence during pregnancy is seldom reported $(4,5)$.

In an earlier report, we speculated that pregnant animals are likely to be more vulnerable to scorpion envenoming, and miscarriages could occur when rats are acutely exposed to lethal doses (6). Moreover, a perturbation of hormone levels (estradiol and progesterone) occurred after scorpion venom injection (6). This suggests that the intrauterine embryo-fetal development 
could be altered. Similarly, post-natal behavioral and neurological alterations and increased body weight were observed after experimental envenoming in gravid rats (7). On the contrary, Barão et al. $(7,8)$, Cruttenden et al. (9) and Dorce et al. (10) found that a single injection of Tityus scorpion venom to 5 or 10 day-pregnant rats did not alter their reproductive parameters, but there was a significant reduction in the litter size; weight of placenta and lungs when dams received a high dose $(1 \mathrm{mg})$ at the $10^{\text {th }}$ day of gestation.

Since scorpion stings provoke various degrees of acute responses, from rapid ones (that last a few hours or days) to severe ones that lead to death, we suggested that several venom components could reach the fetus and probably induce various developmental alterations that remain shadowed in a single scorpion sting. In view of the limited information on such effects, and the unknown venom pharmacology during pregnancy, the aim of the present paper was to investigate fetus development in a repetitive exposure model. Such design was chosen in order to check if the scorpion venom induces any deleterious effects on embryogenesis and fetal growth.

\section{MATERIALS AND METHODS}

\section{Scorpion Venom}

Buthus occitanus tunetanus (Bot), from the Buthidae family, is frequently involved in human envenomings in Tunisia. Its crude venom was kindly offered by the Pasteur Institute of Tunis (Tunisia), obtained by telson electrical milking and extracted following the method of Miranda et al. (11). Briefly, after electrical milking, it was water extracted, freeze dried, rehydrated and stored at $-20^{\circ} \mathrm{C}$ until further use. At the day of the experiment, crude scorpion venom was appropriately diluted with a $0.9 \%$ saline physiologic solution in order to obtain a protein concentration of $300 \mu \mathrm{g} / \mathrm{kg}$. This venom dose is about $1 / 5$ of the LD50 of Bot in pregnant rats (12).

\section{Rat Breeding and Husbandry}

Experiments employed rats because of the known toxicity of venom doses during their pregnancy $(\mathrm{LD} 50=1587 \mu \mathrm{g} / \mathrm{kg})$. Virgin female Wistar rats (6 to 7 weeks old), weighing $150 \pm$ $10 \mathrm{~g}$, were purchased from the Pasteur Institute of Tunis and breaded at our animal house at constant conditions (temperature of 23 to $25^{\circ} \mathrm{C}$, humidity of about $40 \%$ and light/darkness cycle of 14/10 hours). Rats were caged in groups of four per stainless cage. To obtain timed pregnant rats, they were allowed to an overnight mating with ten week-old mature males (one male per four females), after one week of acclimatization to the breeding-room conditions. The day when spermatozoa were detected in the morning (in vaginal smears) was considered the first day of pregnancy. The experimental protocol was in accordance the experimental animal handling guidelines of the Federation of European Laboratory Animal Science (FELASA, 2002).

\section{Study Design and Measurements}

Rats received a daily intra-peritoneal dose of $300 \mu \mathrm{g} / \mathrm{kg}$ of crude Bot venom (five rats) or physiologic saline solution (seven rats), from the $7^{\text {th }}$ to the $13^{\text {th }}$ day of pregnancy. The injected volume was standardized to $1 \mathrm{~mL} / \mathrm{kg}$. The chosen gestation period is considered critical for organogenesis (13). At the $21^{\text {st }}$ day of gestation, rats were deeply anesthetized with diethyl ether, and 2-mL blood samples were collected via cardiac puncture using a heparinized syringe.

The collected blood was immediately transferred into EDTA tube and centrifuged at $3000 \mathrm{rpm}$ for ten minutes. The supernatant plasma was separated, and stored at $-20^{\circ} \mathrm{C}$ until further biochemical analysis. The blood nitrogen urea, glucose, creatinin and total protein were measured using a Technicon RA-XT (Bayer Corporation Diagnstic LTD, Ireland) automated apparatus. AST (aspartate amino- transferase), ALT (alanine amino- trasferase) and cholesterol plasma levels were determined using automated apparatus (Synchron ${ }^{\circledR}$ CX9 PRO, Beckman Coulter France), at the Laboratory of Biochemistry of CHU Habib Bourguiba of Sfax (Tunisia).

During the course of gestation, rat body weight was recorded at the $1^{\text {st }}, 7^{\text {th }}, 13^{\text {th }}$ and the $21^{\text {st }}$ days of pregnancy. After blood collection, rats were euthanized and laparatomized for organ and fetus harvesting, following deep diethyl ether euthanasia. All organs and fetuses were gently removed. Fetuses were kept in wormed $\left(37^{\circ} \mathrm{C}\right)$ physiologic saline solution and dried upon a filter paper before any measurement. Briefly, rats, their fetus organs, and morphometric parameters of placenta were evaluated in order to verify the expected visceral abnormalities. Eyes anomalies were observed in offspring under 
dissecting microscope following eyelid removal. Occipitonasal and tail lengths and chest width of fetuses were measured as indicators of possible skeletal anomalies, using a micrometer of 0.01 $\mathrm{mm}$ accuracy. Fetal tails were considered shorts when their values were under the control mean minus 2 SD.

\section{Statistical Analysis}

All values are expressed as mean $\pm \mathrm{SD}$, medians or percentage of total number. Comparison of values between the two groups of rats was performed using the non parametric MannWhitney test, and differences were considered as significant when $\mathrm{p} \leq 0.05$.

\section{RESULTS}

\section{Rat Parameters}

There were no significant differences between the weights of venom and saline treated rats at the $1^{\text {st }}, 7^{\text {th }}, 13^{\text {th }}$ and $21^{\text {st }}$ days of pregnancy (Figure 1). Data presented in Table 1 showed that repetitive Buthus occitanus tunetanus crude venom administration to pregnant rats

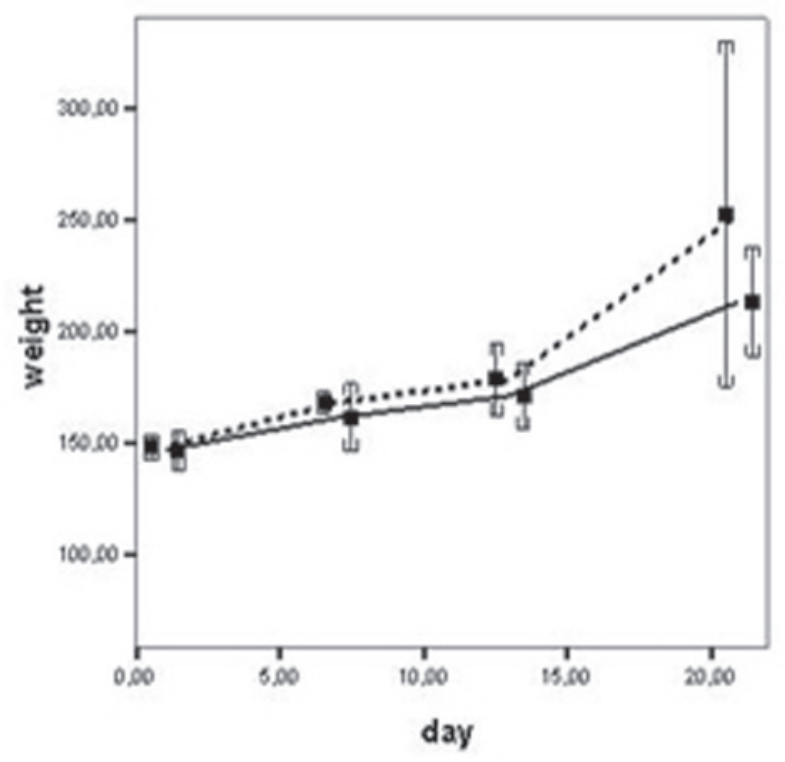

Figure 1. Evolution of rat body weight ( $\mathrm{g}$ ) following repetitive exposure to Buthus occitanus tunetanus venom or saline solution, from the $7^{\text {th }}$ to the $13^{\text {th }}$ day of pregnancy. No significant differences (Mann-Whitney tests) had been checked between venom treated rats (dotted line) and control ones (continuous line). induced a significant increase of their cardiac and pulmonary weights, as well as a significant increase of the fetal relative weight-gain, when compared to saline treated rats. Furthermore, the mean placenta weight per rat was significantly lower $(\mathrm{p}=0.021)$ in venom treated rats $(0.31 \pm$ $0.07 \mathrm{~g} /$ fetus $)$ than in control group $(0.40 \pm 0.01$ $\mathrm{g} /$ fetus).

Other studied morphometric parameters (whole body, total weight gain, gravid uteri, liver, kidneys and spleen weights; and numbers of viable fetuses, number of placentas and resorbed ones, attachment points and dead fetuses) were unchanged in venom and saline treated rats.

Mann-Whitney tests showed constant serum levels for glucose, total protein, cholesterol and creatinine concentrations and aspartate aminotransferase and alanine aminotransferase activities among venom-treated rats and controls. However, there was a significant increase of urea concentration in repetitively envenomed rats (6.9 $\pm 0.6 \mathrm{mmol} / \mathrm{L})$ in comparison to control ones (4.9 $\pm 0.9)$ at $p<0.05$ (Table 1 ).

\section{Outcomes of Fetuses}

Only two deaths of fetuses were recorded in scorpion venom treated rats, but not in controls. The two dead fetuses were from a single rat pregnant with 13 embryos. Three cases of fetal microphtalmia were also observed in venom treated rats and subcutaneous blood clotting was more frequent (56\%) than in control group (10\%) (Table 2).

Figure 2 (A and B) shows morphometric parameters of fetuses. A significant decrease was observed in repetitively envenomed rats in comparison to controls, for the crown-rump lengths, and absolute brain, liver and spleen weights. Such diminishing was also observed among repetitively envenomed rats and control group for relative brain, liver [7.0 \pm 1.8 and 8.7 $\pm 0.8(\%)$, respectively], kidney [0.7 \pm 0.1 and $0.8 \pm 0.1(\%)$, respectively] and spleen weights [0.05 \pm 0.01 and $0.07 \pm 0.01(\%)$, respectively] On the contrary, the relative occipitonasal and tail lengths, and the absolute and relative lung weights were significantly higher in neonates of envenomed rats than those of control ones. The brain/liver indicator was significantly elevated in venom treated rats $(72.2 \pm 23.1)$ when compared to saline treated ones $(57.6 \pm 6.3)$. 
Table 1. Morphometric and biochemical parameters of rats following repetitive Buthus occitanus tunetanus venom exposure from the $7^{\text {th }}$ to $13^{\text {th }}$ day of pregnancy

\begin{tabular}{|c|c|c|c|}
\hline Treatments & Venom & Control & $\mathrm{p}$ values \\
\hline Body weight (j21) (g) & $232 \pm 17$ & $213 \pm 21$ & 0.167 \\
\hline Body weight gain (g) & $84 \pm 13$ & $65 \pm 18$ & 0.167 \\
\hline Gravid uterus weight (g) & $43 \pm 12$ & $48 \pm 6$ & 0.291 \\
\hline Heart weight (g) & $0.5 \pm 0$ & $0.4 \pm 0$ & $0.023^{*}$ \\
\hline Lung weight (g) & $1.9 \pm 0.1$ & $0.9 \pm 0$ & $0.009^{*}$ \\
\hline Liver weight (g) & $7.6 \pm 0.6$ & $7.8 \pm 0.8$ & 0.806 \\
\hline Kidney weight (g) & $1.1 \pm 0.2$ & $0.9 \pm 0$ & 0.080 \\
\hline Spleen weight (g) & $0.35 \pm 0$ & $0.3 \pm 0$ & 0.683 \\
\hline Mean placental weight/rat (g) & $0.30 \pm 0.07$ & $0.40 \pm 0.01$ & $0.021^{*}$ \\
\hline Number of viable fetuses/rat & $9.6 \pm 2.3$ & $10.2 \pm 1.2$ & 0.563 \\
\hline Total number of placenta/rat & $10 \pm 2.8$ & $10.7 \pm 1.8$ & 0.454 \\
\hline Placental resorption (mean number) & $0.8 \pm 0.6$ & $0.7 \pm 0.8$ & 0.360 \\
\hline Mean number of attachment points & $10.4 \pm 2.4$ & $11 \pm 1.7$ & 0.610 \\
\hline Percentage of placental resorption & $7.2 \pm 6.9$ & $6.2 \pm 8.9$ & 0.167 \\
\hline Number of dead fetuses & $2 / 48$ & $0 / 72$ & 0.806 \\
\hline Glucose (mmol/L) & $5.4 \pm 1.07$ & $6.3 \pm 0.88$ & 0.268 \\
\hline Protein (g/L) & $54.3 \pm 6.92$ & $54.1 \pm 4.65$ & 1 \\
\hline Cholesterol (mmol/L) & $1.6 \pm 0.24$ & $1.79 \pm 0.17$ & 0.432 \\
\hline Urea (mmol/L) & $6.97 \pm 0.62$ & $4.94 \pm 0.9$ & $0.018^{*}$ \\
\hline Creatinin $(\mu \mathrm{mol} / \mathrm{L})$ & $38.8 \pm 5.44$ & $38.28 \pm 3.75$ & 0.876 \\
\hline AST (IU/L) & $320.6 \pm 98.8$ & $258.7 \pm 105.9$ & 0.432 \\
\hline ALT (IU/L) & $68.6 \pm 13.9$ & $58.4 \pm 9.9$ & 0.202 \\
\hline
\end{tabular}

All data are reported as mean \pm standard deviation, and were compared using Mann-Whitney non-parametric test; and significant difference ${ }^{*}$ ) was considered at $p<0.05$.

\section{DISCUSSION}

Scorpion envenoming is widely distributed in tropical and subtropical countries. Because of its high frequency, it constitutes a public health problem, especially when appropriate and immediate treatment is not available (4). The toxicological features of such accidents have been extensively studied, but scarce information on their effects on gravid animals is reported (5). In this regard, controversial results of experimental single venom-dose exposure of pregnant rats were observed (7-10). In addition, the lack of information on scorpion venom pharmacology, especially concerning its biodistribution in fetuses, will probably mask the fetal response to the stressor. Given that, this work was designated to evaluate the effect of Buthus occitanus tunetanus venom on pregnant rats and their offspring. To do so, we used a low dose repetitive intoxication model. Our experimental model is thought to permit maternal survival and pregnancy continuation, until the $21^{\text {st }}$ day of gestation (fetal delivery usually occurs at $22 \pm 1$ day of gestation), and the observation of the hypothesized effects on fetal development and growth. 
Table 2. External fetal anomalies observed in offspring of rats repetitively exposed to scorpion venom from the $7^{\text {th }}$ to the $13^{\text {th }}$ day of gestation

\begin{tabular}{c|c|c}
\hline Anomalies & Venom & Control \\
\hline Microphtalmia & $3 / 48(6 \%)$ & $0 / 72(0 \%)$ \\
\hline Short tail length & $16 / 48(33 \%)$ & $3 / 72(4 \%)^{*}$ \\
\hline Subcutaneous blood clotting & $27 / 48(56 \%)$ & $7 / 72(10 \%)^{*}$ \\
\hline
\end{tabular}

The fetal tail was considered short when its length was lesser than the mean control value $-2 \mathrm{SD}$. ${ }^{*}$ significant difference at $\mathrm{p}<0.05$.

The morphometric parameters assessed in rats showed no significant changes between venom-treated rats and their control counterpart concerning whole body weight. This could indicate the mild effects of the repetitive exposure to such small dose of venom on maternal metabolism. However, there was a significant increase in the absolute heart and lungs weights, when compared to control.

Scorpion envenoming triggers edema and plasma extravasation through a mechanism that implicates renal filtration changes, as expected by the observed urea concentration increase (1417). This will inevitably enhance the gestational physiologic edema and thereby contributes to the increased size of maternal organs (18). Changes in blood urea content may also reveal pathological alterations in fetuses (19).

There was no significant difference in the number of viable offspring between the two groups. Interestingly, the repetitive injection of venom induced a slight decrease in placental weight, which commonly leads to embryo-fetal growth disorders $(20,21)$. While fetuses of venom
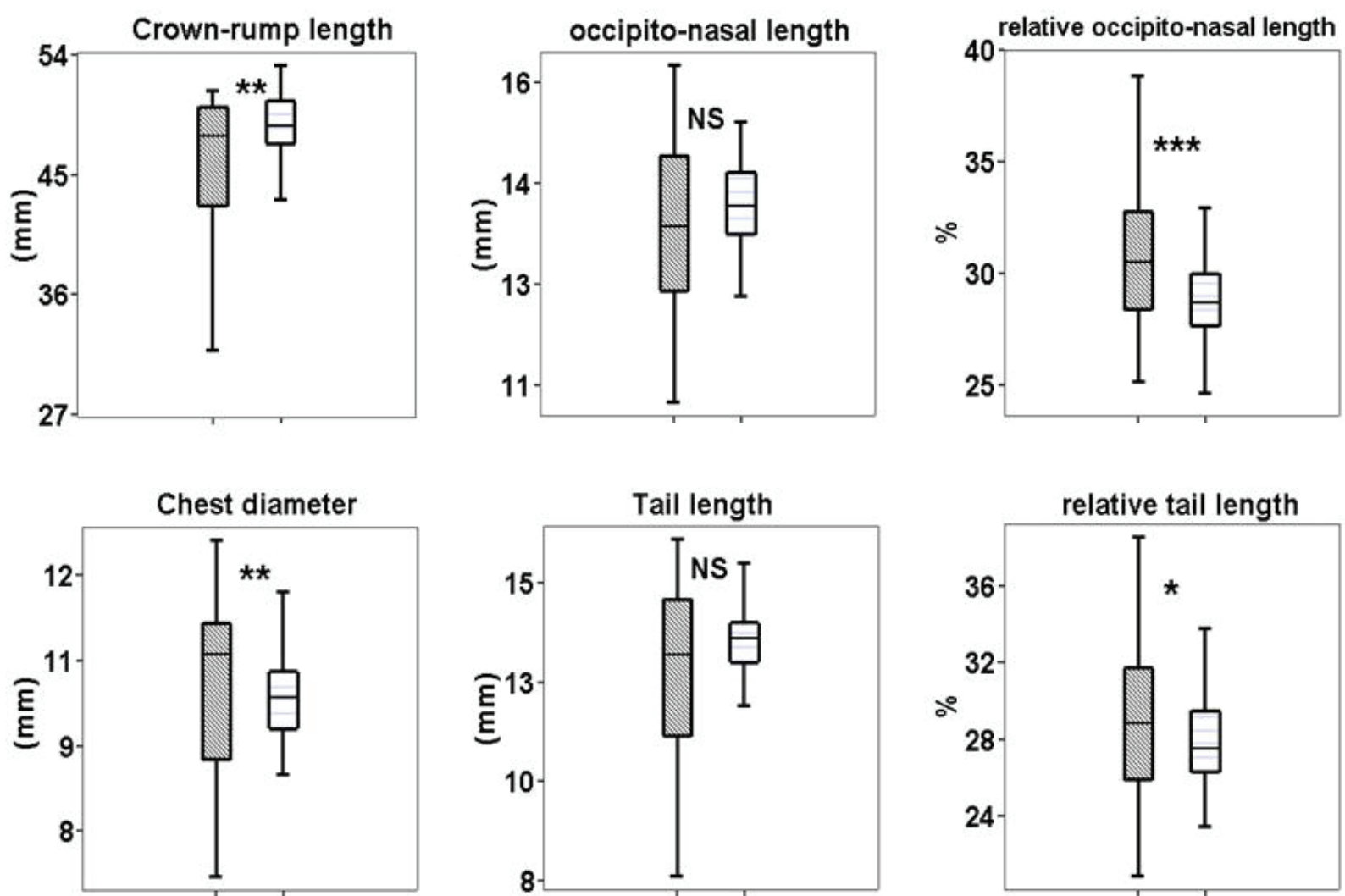

Figure 2. External body sizes of fetuses from pregnant rats treated with a daily dose of $300 \mu \mathrm{g} / \mathrm{kg}$ of Buthus occitanus tunetanus venom (striped boxes) or $1 \mathrm{~mL} / \mathrm{kg}$ of saline solution (white boxes), from the $7^{\text {th }}$ to the $13^{\text {th }}$ day of gestation ( $\longrightarrow$ median, $\square 25 \%-75 \%, \square$ min.-max.). 

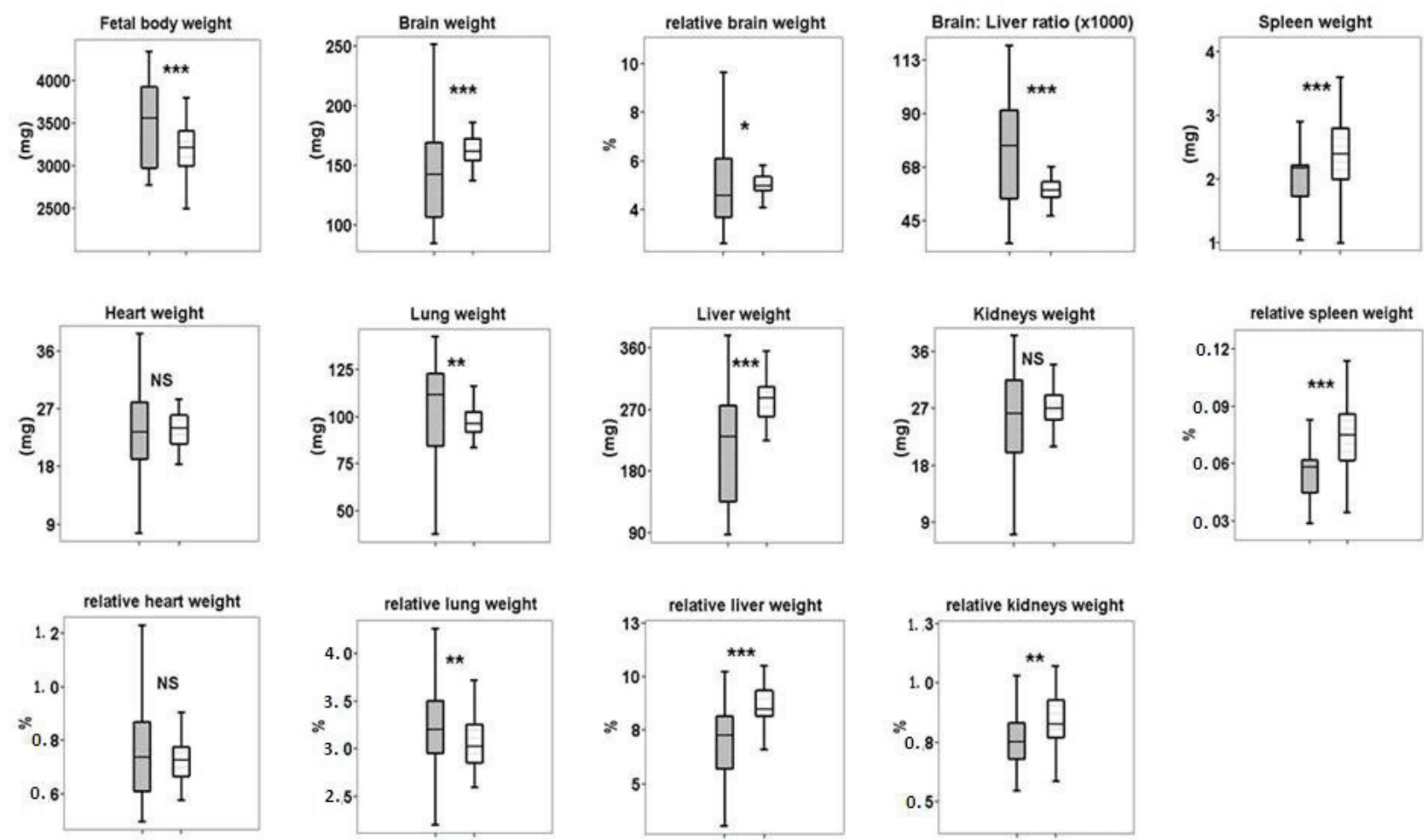

Figure 3. Viscera weights of fetuses from pregnant rats treated with a daily dose of $300 \mu \mathrm{g} / \mathrm{kg}$ of Buthus occitanus tunetanus venom (striped boxes) or $1 \mathrm{~mL} / \mathrm{kg}$ of saline solution (white boxes), from the $7^{\text {th }}$ to the $13^{\text {th }}$ day of gestation ( - median, $25 \%-75 \%, \square$ min.-max.).

treated-rats had heavier body weights, the size of many of their vital organs significantly changed in comparison to control ones. In fact, the repetitive maternal exposure to scorpion toxins provoked growth restriction in the brain, liver, kidneys and spleen. On the contrary, increase in fetal lung size was observed after venom treatment.

In exception for the whole body and lung weights, other organ disturbances had not been observed following a single injection of scorpion venoms during pregnancy (7-10). Ismail et al. (22) showed that chronic maternal exposure to Androctonus amoreuxi venom lead to intrauterine growth impairment. The contradiction between their findings and ours may be attributed to differences in the experimental design. Furthermore, differences in scorpion species and their venom composition may explain the divergence in the results (1). Commonly, this disordered intrauterine growth of fetus organs can be justified by two mechanisms: a defective placental function resulting from placenta narrowness and perfusion alterations; and maternal hypertension and metabolic disorders $(20,21,23,24)$. As the repetitive venom injection induces intermittent hypertension, it will indirectly results in fetal growth disorder (25). Additional histopathological examinations would be helpful to for further analysis.

In addition to the recorded anomalies (microphtalmia and subcutaneous blood clots), an unexpected significant shortage of neonate crown-rump length and slight decrease in absolute head and tail lengths were observed following maternal envenoming. Similarly, in studies on embryo-toxic effects of acute maternal exposure to single dose venom, Barão et al. (7) showed that the skull bone was affected. Moreover, it had been proven that Heterometrus bengalensis scorpion venom influences bone remodeling (26). Thus, it suggested that scorpion envenoming during pregnancy may cause prenatal structural anomalies in skeleton formation.

The increased size of brain and liver indicates intrauterine growth disorder, as proposed in diabetic embryopathy (27). While these disturbances are not shown in normal conditions of scorpion stings, experimental studies indicated that neonates have altered postnatal behavior implying the altered neurological function (7-9).

Furthermore, it is important to note that scorpion sting may provoke an additive stress, 
such as pain and anxiety that could participate in intrauterine growth restriction and postnatal neurobehavioral pathologies $(1,28,29)$. Besides, prenatal stressing factors are suggested as indirect causers of the observed offspring outcomes.

Such finding put forward the necessity of pediatric survey following maternal scorpion stings. Throughout the literature, there are no concise investigations of scorpion toxin pharmacology during conception. Because of that, we speculate the indirect embryotoxic effects of this envenoming which could originate from the excessive release of reactive oxygen species, especially in fetal tissues, and the maternal hemodynamic alteration that are implicated in embryo-fetal development and growth perturbations (1, 6, 30-33).

Our findings suggest that scorpion venom, when repetitively injected during pregnancy, can induce intrauterine anomalies of fetal growth and development. To appropriately disclose this matter in normal scorpionism accidents, it is proposed to investigate the venom pharmacodynamics and pharmacokinetics during conception and postnatal period.

\section{ACKNOWLEDGMENTS}

The authors greatly thank Dr Mion Georges (Ecole du Val-de-Grâce, France) for their advices in running the statistical analysis and penning the manuscript.

\section{COPYRIGHT}

(C) CEVAP 2012

\section{SUBMISSION STATUS}

Received: May 14, 2012.

Accepted: July 10, 2012.

Abstract published online: July 10, 2012.

Full paper published online: August 31, 2012.

\section{CONFLICTS OF INTEREST}

The authors declare no conflicts of interest.

\section{ETHICS COMMITTEE APPROVAL}

The present study was approved by a committee of senior researchers from the Medical School of Sfax, Sfax, Tunisia, and the Pasteur Institute, Tunis, Tunisia.

\section{CORRESPONDENCE TO}

Ben-Nasr Hmed, Laboratory of Pharmacology, Sfax Faculty of Medicine, Rue Magida Boulila 3029, Sfax, Tunisia. Phone: 21674247 036. Fax: 21674246 217. Email: hmedbnasr@gmail.com.

\section{REFERENCES}

1. Goyffon M. Le scorpionisme. Rev Fr Lab. 2002;342:418.

2. Nassar AY, Abu-Sinna G, Rahim SA. Effect of a bradykinin potentiating fraction, from venom of the Egyptian scorpion, Buthus occitanus, on the ovaries and endometrium of mice. Toxicon. 1990;28(5):52534 .

3. el-Saadani MA. A scorpion venom peptide fraction induced prostaglandin biosynthesis in guinea pig kidneys: incorporation of 14C-linoleic acid. J Biochem. 2004;135(1):109-16

4. ChippauxJP, Goyffon M. Epidemiology of scorpionism: a global appraisal. Acta Trop. 2008,107(2):71-9.

5. Langley RL. A review of venomous animal bites and stings in pregnant patients. Wilderness and Environ Med. 2004;15(3):207-15.

6. Ben Nasr H, Serria H, Chaker S, Riadh B, Zouheir S, Kamel J, et al. Some biological effects of scorpion envenomation in late pregnant rats. Exp Toxicol Pathol. 2009,61(6):573-80.

7. Barão AA, Bellot RG, Dorce VA. Developmental effects of Tityus serrulatus scorpion venom on the rat offspring. Brain Res Bull. 2008;76(5):499-504.

8. Barão AA, Nencioni AL, Dorce AC. Embryotoxic effects of maternal exposure to Tityus serrulatus scorpion venom. J Venom Anim Toxins incl Trop Dis. 2008;14(2):322-37.

9. Cruttenden K, Nencioni AL, Bernardi MM, Dorce VA. Reproductive toxic effects of Tityus serrulatus scorpion venom in rats. Reprod Toxicol. 2008;25(4):497-503.

10. Dorce AL, Bellot RG, Dorce VA, Nencioni AL. Effects of prenatal exposure to Tityus bahiensis scorpion venom on rat offspring development. Reprod Toxicol. 2009;28(3):365-70.

11. Miranda F, Kupeyan C, Rochat H, Rochat C, Lissitzky S. Purification of animal toxins: Isolation and characterization of eleven neurotoxins from the venoms of the scorpions Androctonus australis hector, Buthus occitanus tunetanus and Leiurus quinquestriatus quinquestriatus. Eur J Biochem. 1970;16(3):514-23.

12. Hmed BN, Serria HT, Selma C, Sahnoun S, Tarek R, Mondher $\mathrm{K}$, et al. In vivo effects of Buthus occitanus tunetanus and Androctonus australis garzoni scorpion venoms in pregnant and non pregnant rats. J Venom Anim Toxins incl Trop Dis. 2008;14(2):366-71.

13. Molenberghs G, Geys H, Buyse M. Evaluation of surrogate endpoints in randomized experiments with mixed discrete and continuous outcomes. Stat Med. 2001;20(20):3023-38. 
14. de Souza Alves R,do Nascimento NR, Barbosa PS, Kerntopf MR, Lessa LL, de Sousa CM, et al. Renal effects and vascular reactivity induced by Tityus serrulatus venom. Toxicon. 2005,46(3):271-6.

15. Chen B, Zhuo X, Wang C, Ji Y. Asian scorpion Bmk venom induces plasma extravasation and thermal hyperalgesia in the rat. Toxicon. 2002;40(5):527-33.

16. Coelho FM, Pessini AC, Coelho AM, Pinho VS, Souza DG, Arantes EC. Platelet activating factor receptors drive CXC chemokine production, neutrophil influx and edema formation in the lungs of mice injected with Tityus serrulatus venom. Toxicon. 2007;50(3):420-7.

17. Corrêa MM, Sampaio SV, Lopes RA, Mancuso LC, Cunha OA, Franco JJ, et al. Biochemical and histopathological alterations induced in rats by Tityus serrulatus scorpion venom and its major neurotoxin tityustoxin-I. Toxicon. 1997;35(7):1053-67.

18. Daniel SS, Stark RI, Tropper PJ, James LS. Amniotic fluid composition in the fetal lamb with intrauterine growth restriction. Am Obstet Gynecol. 1999;180(3 Pt 1):703-10.

19. Thurlow RW, Brace RA. Swallowing, urine flow, and amniotic fluid volume responses to prolonged hypoxia in the ovine fetus. Am J Obstet Gynecol. 2003;189(2):601-8.

20. Blecker OP, Buimer M, van der Post JA, van der Veen F, Ted (g.j.) Kloosterman: on intrauterine growth. The significance of prenatal care. Studies on birth weight, placental weight and placental index. Placenta. 2006;27(11-12):1052-4.

21. Gude NM, Roberts CT, Kalionis B, King RG. Growth and function of the normal human placenta. Thromb Res. 2004;114(5-6):397-407.

22. Ismail M, Ellison AC, Tilimsany AK. Teratogenicity in the rat of the venom from the scorpion Androctonus amoreuxi (Aud. \& Sav.). Toxicon. 1983;21(2):177-89.

23. Barr M Jr. Correlates of prenatal visceromegaly. Am J Med Genet. 1998;79(4):249-52.

24. Ness RB, Sibai BM. Shared and disparate components of the pathophysiologies of fetal growth restriction and preeclampsia. Am J Obstet Gynecol. 2006;195(1):40-9.
25. Tranquilli AL, Giannubilo SR, Dell'Uomo B, Corradetti A. Prediction of gestational hypertension or intrauterine fetal growth restriction by midtrimester 24-h ambulatory blood pressure monitoring. Int J Gynaecol Obstet. 2004,85(2):126-31.

26. Gomes A, Haldar S, Giri B, Mishra R, Saha A, Dasgupta S, et al. Experimental osteoporosis induced in female albino rats and its antagonism by Indian black scorpion (Heterometrus bengalensis C.L.Koch) venom. Toxicon. 2009;53(1):60-8.

27. Mitchell ML. Fetal brain liver weight ration as a measure of intrauterine growth retardation: analysis of 182 stillborn autopsies. Mod Pathol. 2001;14(1):14-9.

28. Anand KJ, Coskun V, Thrivikraman KV, Nemeroff $\mathrm{CB}$, Plotsky PM. Long-term behavioural effects of repetitive pain in neonatal rat pups. Physiol Behav. 1999;66(4):627-37.

29. Van den Bergh BR, Mulder EJ, Mennes M, Glover V. Antenatal maternal anxiety and stress the neurobehavioural development of the fetus and child: links and possible mechanisms. A review. Neurosci Biobehav Rev. 2005;29(2):237-58.

30. Dousset E, Carrega L, Steinberg JG, Clot-Faybesse O, Jouirou B, Sauze N, et al. Evidence that free radical generation occurs during scorpion envenomation. Comp Biochem Physiol C Toxicol Pharmacol. 2005;140(2):221-6.

31. Ornoy A. Embryonic oxidative stress as a mechanism of teratogenesis with special emphasis on diabetic embryopathy. Reprod Toxicol. 2007;24(1):31-41.

32. Xu DX, Chen YH, Zhao L, Wang H, Wei W. Reactive oxygen species are involved in lipopolysaccharide induced intrauterine growth restriction and skeletal development retardation in mice. Am J Obstet Gynecol. 2006;195(6):1707-14.

33. Schwartz JE, Kovach A, Meyer J, McConnel C, Iwamato HS. Brief, intermittent hypoxia restricts fetal growth in Sprague Dawley rats. Biol Neonate. 1998;73(5):3139. 